\title{
Coloured and low conductive fabrics by in situ laccase-catalysed polymerization
}

\author{
Jing Su ${ }^{\mathrm{a}, \mathrm{b}}$, Jennifer Noro ${ }^{\mathrm{b}}$, Jiajia Fu ${ }^{\mathrm{a}}$, Qiang Wang ${ }^{\mathrm{a}}$, Carla Silva ${ }^{\mathrm{b}}$, Artur Cavaco-Paulo ${ }^{\mathrm{a}, \mathrm{b}, *}$ \\ ${ }^{a}$ International Joint Research Laboratory for Textile and Fiber Bioprocesses, Jiangnan University, Wuxi, 214122, China \\ ${ }^{\mathrm{b}}$ Centre of Biological Engineering, University of Minho, Campus de Gualtar, 4710-057, Braga, Portugal
}

\section{A R T I C L E I N F O}

\section{Keywords:}

Laccase-catalysed polymers

Fabrics

Colouration

Conductivity

\begin{abstract}
A B S T R A C T
Coloured and conductive fabrics were obtained through "in situ" laccase polymerization of catechol and $p$ phenylenediamine under high-pressure homogenization. Both monomers, catechol and p-phenylenediamine, were polymerized by different laccase forms, namely native, PEGylated and Epoxy-PEGylated. All the catalysts were placed inside a textile fabric bag which served simultaneously as enzyme support and as substrate for coating with the newly produced polymers. The PEGylated laccase forms gave rise to a higher amount of oligomers/polymers and higher colouration level of polyethylene terephthalate (PET), cotton and wool fabrics compared to native laccase. Both functional polymers were able to confer conductivity to the substrates however in a different extent. Fabrics coated with poly(p-phenylenediamine) present higher conductivity, rather due to its polymerized structure than to the amount of polymer produced by enzyme catalysis. Herein a green approach was presented to produce polyphenols with increased fixation onto different textile substrates. These substrates reach high levels of colouration and good fastness behaviour after washing.
\end{abstract}

\section{Introduction}

Phenolic and aromatic amine compounds like catechol and $p$-phenylenediamine have been widely used as industrial reagents to produce pharmaceutical products, cosmetics, dyes, rubber and plastics [1,2]. These industrial activities produce polluted wastewater containing these compounds which may accumulate in the soil, ground and surface water, when released into the environment [3]. Therefore, their removal from wastewater is of extreme importance in the referred areas. Oxidoreductive enzymes have been explored to catalyse the transformation of the toxic phenols and aryldiamines in valuable products for diverse applications [4-6]. Among the different existing oxidant enzymes, laccases (benzenediol: oxygen oxidoreductases; EC 1.10.3.2) are considered as one of the most important ones since they can catalyse the oxidation of phenol/amine compounds and their derivatives. Laccase-assisted reactions take place under mild conditions in the absence of toxic reagents and rarely cause the formation of by-products. This oxidation ability has been presented as a promising route to obtain ecological functional oligomers and polymers with varied functions such as dyeability, antioxidant activity, conductivity and other properties, for application in several fields [7-10]. Polymeric dyes are coloured polymers synthesized through controlled reactions, such as monomer polymerization and macromolecule modification, to bring chromophore to the main or side polymers [11]. Their higher colouration ability and colour fastness make them good alternatives for the dyeing of textile fibres since traditional dyes involve high energy consumption and wastewater drawbacks. Recently, laccases have been investigated for the biosynthesis of bio-colourants and decolouration of synthetic dyestuffs in the dyeing industry with some researches focusing on the enzymatic colouration of natural fibres. Shin et al. proved that laccase had the ability to dye wool fabric after padding with hydroquinone [12]. Tzanov et al. also confirmed that laccases could be applied to wool dyeing [13]. Later, Kim et al. explored the ability of laccase for the colouration of cotton and flax, aiming fibre surface modification [14-16]. Despite the promising results obtained the washing and rubbing fastness results were not adequate, due to the low substantivity of the new polymers onto the fibres surface. Deeper studies including the use of different devices for the in situ polymerization of the phenolics would help to overcome the substantivity problems associated to this green colouration method.

Besides colouration, the new polymers supply generally additional properties to the substrates on which they are applied, namely electrical conductivity, antimicrobial and antioxidant behaviour, among others [17-19]. Electrical conductivity conferred by these polymers is the

\footnotetext{
* Corresponding author at: Centre of Biological Engineering, University of Minho, Campus de Gualtar, 4710-057, Braga, Portugal. International Joint Research Laboratory for Textile and Fiber Bioprocesses, Jiangnan University, Wuxi 214122, China.

E-mail address: artur@deb.uminho.pt (A. Cavaco-Paulo).
} 
result of the delocalization of $\pi$-electrons in a conjugated system. The presence of electric charges in their molecular structure is capable of translocating along the polymer chains without involving other conducting materials such as metals or graphite [19]. In literature one can find already a considerable number of works related to the enzymatic approaches for the production of conductive products. Recently Zhang et al. successfully developed an enzymatic approach for dyeing wool fabrics with special $\mathrm{pH}$-responsive, colour-changing and conductive properties, via in-situ polymerization of 2,5-diaminobenzenesulfonic acid (DABSA) by laccase from Trametes versicolor [20]. High-redoxpotential laccase, isolated from Aspergillus, was used by the same researchers as a biocatalyst in the synthesis of conducting polyaniline/ lignosulfonate (PANI-ES-LGS) complex using atmospheric oxygen as the oxidizing agent [21]. Salas et al. found the optimal enzymatic conditions for the polymerization of aniline using a commercial Trametes villosa laccase [22]. All these studies involve mostly the use of water bath reactors to conduct the polymerization and colouration. In previous studies, we explored the role of high energy environments on the enzymatic polymerization of catechol. Our findings suggested that the use of devices like high-pressure homogenizers (HPH) for the enzymatic polymerization of phenolics greatly improve the conversion rates and polymerization degrees [23]. High-pressure homogenization is a technology generally applied to reduce the particles size using continuous or semi-continuous pressures between 60-400 M P [24]. High-pressure homogenizer pump a fluid through a narrow gap valve (the heart of homogenizing equipment) using high pressure intensifiers, which increases its velocity to a great extent, resulting in depressurization with consequent cavitation and high shear stress [25].

The main objective of this study is to colourize and confer electrical conductivity to textile fabrics through a green in situ polymerization of catechol and $p$-phenylenediamine under high pressure homogenization. Within this methodology we foresee to overcome the substantivity problems associated with the biocolouration of fibres with poly(phenolics), where low fastness levels are normally obtained. Laccase was used as the biocatalyst for the biosynthesis and in situ coating of textile fabrics (polyethylene terephthalate, cotton and wool) with the newly polymers formed. The polymerizations were conducted with different laccase forms, namely native laccase, PEGylated laccase and PEGylated laccase immobilized onto an epoxy-resin. The latter catalyst form was deposited inside of textile fibre bags (polyester, cotton and wool) during processing, as well as the other forms for control purpose. The ability of the produced polymers as dyestuff and conductive additives was evaluated. The conversion rates of the polymerization reactions were assessed by ${ }^{1} \mathrm{H}$ NMR spectroscopy and gravimetrically.

\section{Material and methods}

\subsection{Materials and equipment}

Laccase from Myceliophthora thermophila was supplied by Novozymes, Denmark. Catechol, p-phenylenediamine, poly(ethylene glycol) methyl ether and sodium carbonate were purchased from Sigma Aldrich, Spain. Deuterated dimethyl sulfoxide was obtained from Cortecnet, France.

The fabrics used for the study were supplied by a textile company with the following characteristics: polyethylene therephthalate (PET): $96 \mathrm{~g} / \mathrm{m}^{2} ; 24 * 40 / \mathrm{cm}^{2}$, cotton: $65 \mathrm{~g} / \mathrm{m}^{2} ; 35 * 40 / \mathrm{cm}^{2}$ and wool: $420 \mathrm{~g}$ / $\mathrm{m}^{2} ; 15 * 15 / \mathrm{cm}^{2}$.

The high-pressure homogenizer used for the experiments was the EmulsiFlex-C3 (ATA Scientific, Taren Point NSW, Australia) with a constant flow-through capacity of $3 \mathrm{~L} / \mathrm{hr}$ with the ability to process $10 \mathrm{~mL}$ samples; the homogenizing pressure is adjustable between 500 and 30,000 psi or 35 and 2000 bar; the flow rate is independent of pressure.

\subsection{Methods}

\subsubsection{PEGylation of laccase}

Laccase from Myceliophthora thermophila was PEGylated as previously reported [26] using the procedure of Daly et al. [27]. Briefly, $14.0 \mathrm{~mL}$ of $12 \mathrm{mg} / \mathrm{mL}$ laccase were reacted with $20 \mathrm{kDa}$, poly(ethylene glycol) methyl ether at $\mathrm{pH}=5,10 \mathrm{mM}$ sodium phosphate buffer with $20 \mathrm{mM}$ sodium cyanoborohydride. A control reaction without $\mathrm{mPEG}$ was also conducted in every experiment. The reactions were stirred rapidly for $17 \mathrm{~h}$ at $4{ }^{\circ} \mathrm{C}$. After $10 \mathrm{~min}$ of mixing, the reactants were completely dissolved, and an aliquot (namely time $0 \mathrm{~h}$ ) was taken, as well as at each time point of reaction. These samples were ultrafiltrated using a $30 \mathrm{kDa}$ cellulose membrane mounted in an ultrafiltration apparatus. Then, the activity of laccase was measured against ABTS according to the methodology described by Childs and Bardsley [28].

\subsubsection{Immobilization of PEGylated laccase onto epoxy resin supports}

The immobilization of PEGylated laccase onto epoxy methacrylate resins (Purelite Lifetech ECR enzyme immobilization resins: 300-600 ̊) was conducted as follows: $2 \mathrm{mg} / \mathrm{mL}$ PEGylated laccase in $0.5 \mathrm{M}$ acetate buffer ( $\mathrm{pH} 5.0$ ) were mixed with epoxy methacrylate $(50 \mathrm{mg} / \mathrm{mL})$ and then stirred for $48 \mathrm{~h}$ at $4{ }^{\circ} \mathrm{C}$. The powder was then washed several times with water by centrifugation and dried under vacuum.

\subsubsection{Enzymatic-assisted polymerization of catechol and p-} phenylenediamine

The biotransformation of catechol and $p$-phenylenediamine into their functional polymers was conducted in acetate buffer ( $\mathrm{pH} 5$ ) through high-pressure homogenization. Catechol and p-phenylenediamine polymerization were processed by incubating $50 \mathrm{mM}$ of monomer in different solutions: a) $100 \mathrm{U} / \mathrm{mL}$ native laccase and b) $100 \mathrm{U} / \mathrm{mL}$ PEGylated laccase, c) $100 \mathrm{U} / \mathrm{ml}$ epoxy-PEGylated laccase, in acetate buffer $(\mathrm{pH}=5)$. The immobilized enzyme was confined in a polyethylene bag and placed in the sample receptor of the high-pressure homogenizer. This receptor is fed with the fluid that undergoes homogenization inside the device and crosses the fabric sample, at this stage, with reduced pressure. The other enzyme forms were also placed inside the bag for control purposes. Afterwards the monomer solution was added, and the homogenization proceed for $2 \mathrm{~h}$ (corresponding to 360 homogenization cycles). The starting temperature was set to $40^{\circ} \mathrm{C}$ and the temperature was monitored during processing to follow the inherent increase due to high-energy device processing. Further the polymer powder was collected from the HPH device by dissolution with dimethyl sulfoxide to solubilize the insoluble polymers and posteriorly dried under vacuum for characterization.

Controls comprising fabric samples incubated only with laccase were also conducted in order to evaluate the influence of the catalyst colour on the fabrics after homogenization.

\subsubsection{Polymers characterization by ${ }^{1} H \quad N M R$ spectroscopy and gravimetrically}

The polymers produced were collected from solution and characterized by ${ }^{1} \mathrm{H}$ NMR. For this the precipitates obtained after washing and centrifugation were dissolved in deuterated solvent, DMSO- $\mathrm{d}_{6}$ for ${ }^{1} \mathrm{H}$ NMR evaluation. The spectra were acquired in a Bruker Avance III $400(400 \mathrm{MHz})$ using the peak solvent as internal reference.

The weighing of the final polymer powder took place by weighting the powder collected from the final solution and the weight of polymer on the coated fabric samples (the weight of samples after coating is subtracted to the initial weight in dry conditions).

\subsubsection{Wettability evaluation: water contact angle and water drop test}

Dataphysics equipment using OCA software with video system (Dataphysics, Filderstadt, Germany) for the capturing of images in static and dynamic modes was used for the measurement of contact angle of the water drop in the non coated and in the coated fabrics, at 


\section{a. catechol}
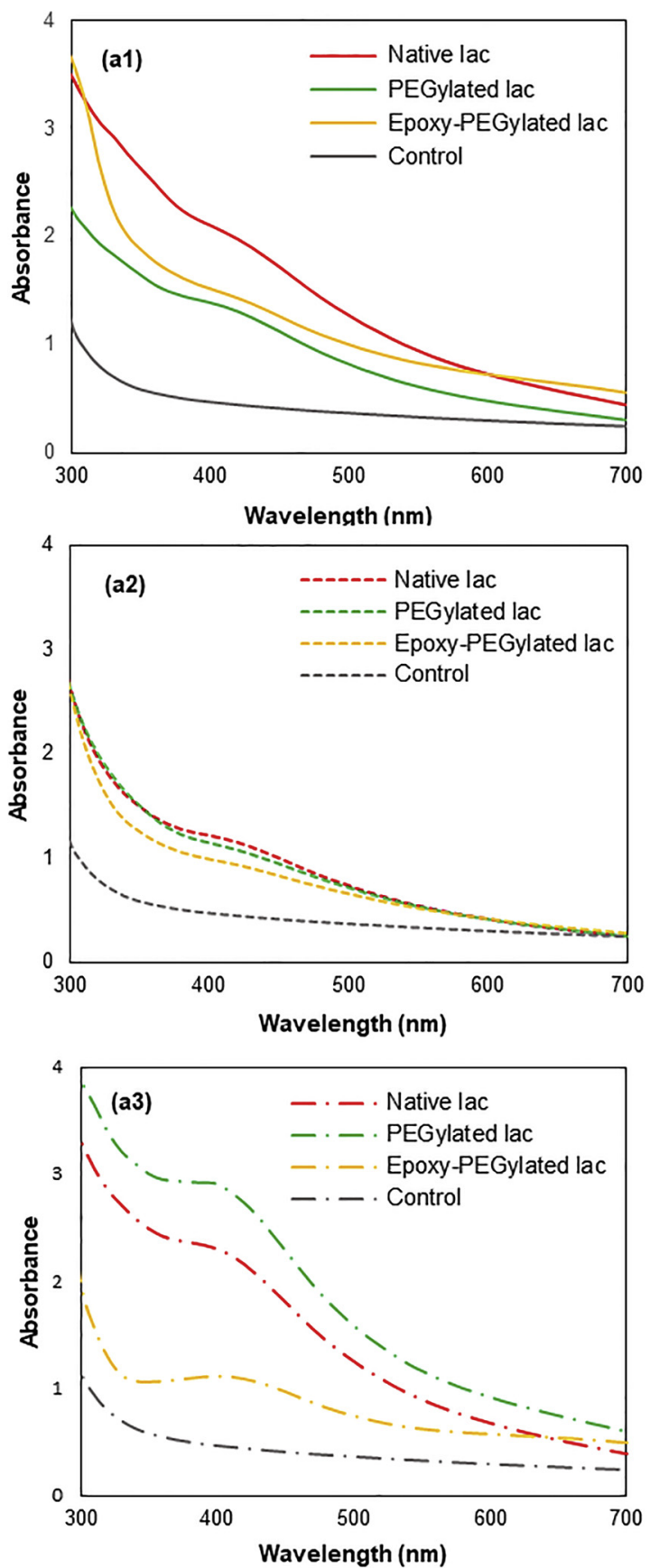

b. p-phenylenediamine
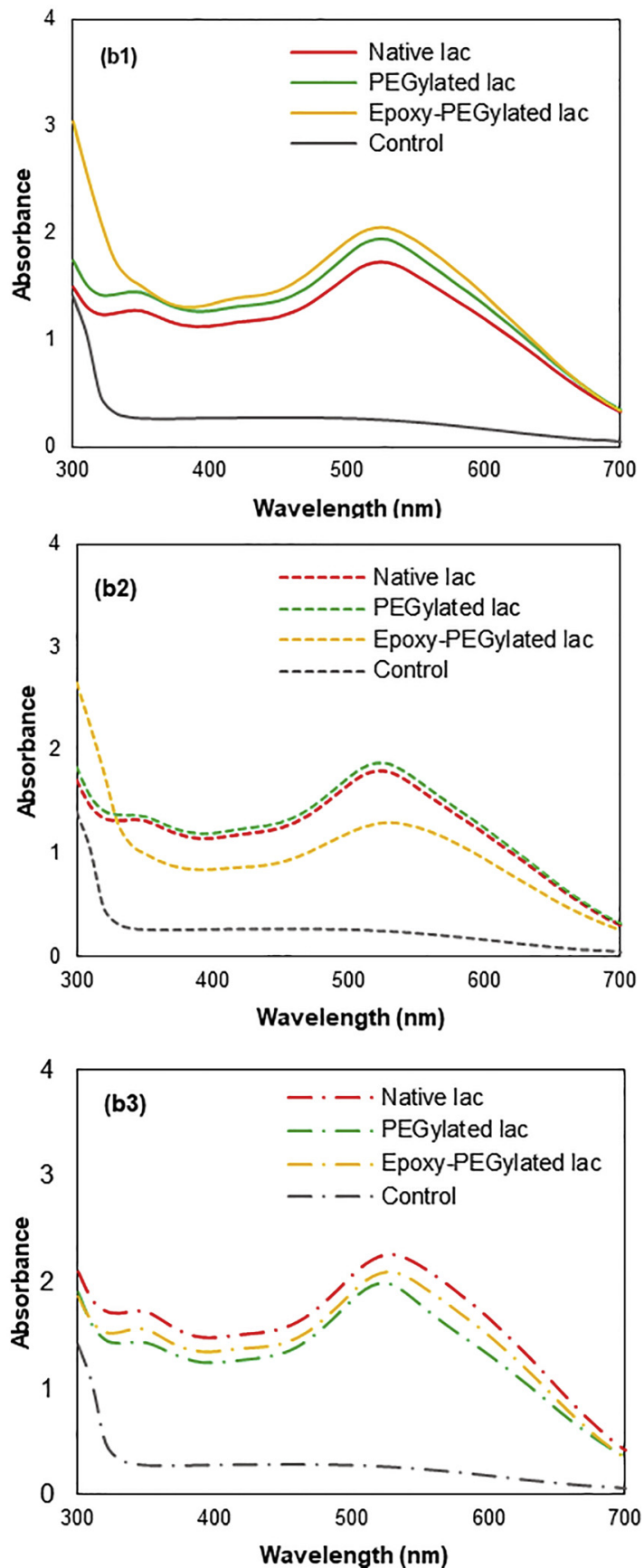

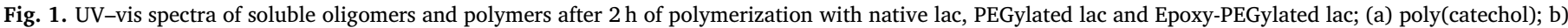

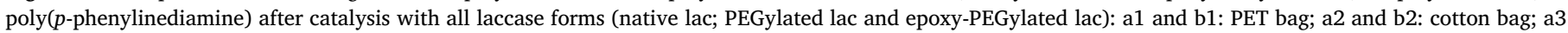
and b3: wool bag; the control corresponds to a monomer solution without addition of enzyme.

room temperature. Contact angles were measured after $15 \mathrm{~s}$ and the data were obtained from the averages of measurements taken from ten different points on the surface of each sample. The volume of the water droplet was set as $5 \mu \mathrm{L}$ using a Hamilton $500 \mu \mathrm{L}$ syringe type and the model select to measure the water contact angle was the Ellipse-fitting model (evaluation of the outline by fitting an ellipse).

The degree of wettability of the coated samples was evaluated by means of the water drop test according to AATCC standard method [29]. The wetting time was determined by placing a drop of distilled water on the stretched fabric sample $(2 \mathrm{~cm} \times 2 \mathrm{~cm})$ from a burette held $1 \mathrm{~cm}$ from the fabric. The time of disappearance of the water-mirror on the surface (the time for the water drop to lose its reflective power) was measured as the wetting time. This procedure was applied to both uncoated and coated fabrics.

\subsubsection{Scanning electron microscopy}

All the fabric samples were added to aluminium pin stubs with electrically conductive carbon adhesive tape (PELCO Tabs $^{\mathrm{TM}}$ ), with the excess removed using compressed air. Samples were coated with $2 \mathrm{~nm}$ of $\mathrm{Au}$ for improved conductivity. The aluminium pin stub was then placed inside a Phenom Standard Sample Holder, and different points for each sample were analysed for elemental composition. The samples were characterized using a desktop scanning electron microscope (SEM) coupled with energy-dispersive X-ray spectroscopy (EDS) 
analysis (Phenom ProX with EDS detector (Phenom-World BV, Netherlands)). All results were acquired using the ProSuite software integrated with Phenom Element Identification software, allowed for the quantification of the concentration of the elements present in the samples, expressed in either weight or atomic concentration.

\section{3. $K / S$ evaluation of coloured fabrics}

The colour strength $(k / s$ values) was evaluated by using a Datacolour apparatus at standard illuminant D65 with the KubelkaMunk equation (Eq. 1), in which $K$ is the absorbance coefficient, $S$ is the scattering coefficient and $R$ is the reflectance ratio.

Since not all the samples presented the same maximum wavelength, the data were presented as the sum of all $K / S$ values obtained in the wavelength range $400-700 \mathrm{~nm}$ (checksum $K / S$ ) at the measuring intervals of $10 \mathrm{~nm}$. The measurements were done in triplicate and the data presented are the mean values of these measurements.

$\frac{K}{\mathrm{~S}}=\frac{(1-\mathrm{R})^{2}}{2 \mathrm{R}}$

Colour difference was qualitatively evaluated using the software Spectraflash 600 from Datacolour using the $K / S$ values for difference evaluation and attributing values from 1-5.

\subsection{Conductivity measurement of coated fabrics}

Electrical conductivities were measured with a Fluke 123 Scopmeter (20Mhz) using two -point probe technique placing them under a predefined distance between. The conductivity was calculated according to the following equation:

Conductivity $(\sigma)=1 / \rho\left(\mathrm{S}_{\mathrm{cm}}{ }^{-1}\right)$,

obtained from the calculation $\mathrm{R}=\rho(\mathrm{L} / \mathrm{A})$, where $\mathrm{R}$ : resistance in ohm.cm; $\rho$ : resistivity $(\mathrm{cm})$; L: distance between electrodes $(6 \mathrm{~mm})$; A: area of material section $(6 * 0.5 \mathrm{~mm})$.

\section{Results and discussion}

\subsection{Catechol and p-phenylenediamine polymerization}

The biotransformation of catechol and p-phenylenediamine into their functional polymers was conducted in acetate buffer $(\mathrm{pH} 5)$ through high-pressure homogenization and followed by UV-vis spectroscopy (Fig. 1). Observing both spectra sets one can infer a different spectral behaviour during polymerization for catechol and p-phenylenediamine. During laccase-assisted polymerization of catechol under high-pressure homogenization, all the reaction mixtures changed from colourless to dark brown. The spectra of the soluble oligomers present a typical peak around $300 \mathrm{~nm}$ and, due to molecular arrangements inherent to polymer formation, the peak intensity increases. At the same time, a new peak at around $430 \mathrm{~nm}$ appears confirming polymerization. The spectra of $p$-phenylenediamine presents a different feature. The colourless monomer presents a typical spectrum with a peak at around $300 \mathrm{~nm}$. As the oxidation starts ( $\approx 15 \mathrm{~min}$.) the solution gets blue-violet and a new absorption band is developed at around $520-530 \mathrm{~nm}$ as well as a new shoulder at around $350 \mathrm{~nm}$. After $2 \mathrm{~h}$ of oxidation, the soluble oligomers maintain the described spectra (Fig. 1) and some precipitates can be observed at the bottom of the solution container, corresponding to the insoluble oligomers. Despite the different spectra observed, both events are governed by an increase in the UV-vis absorption intensity indicating a greater degree of $\pi$-conjugation correlated with the occurrence of polymerization [30].

These preliminary findings seem to point out that PEGylated and Epoxy-PEGylated laccase forms are more prone to polymerize both catechol and $p$-phenylenediamine. However, the tendency is not the same for all the samples since depending on the fibre used as enzyme container, the amount of soluble oligomers available for detection is different. These is discussed further on the spectra estimation discussion.

The ${ }^{1} \mathrm{H}$ NMR of poly(catechol) (Figure S1-A) shows that after polymerization, the $\mathrm{OH}$ peak (proton c) from the catechol starting material disappeared or decreased in intensity, indicating the polymerization of catechol by the hydroxyl groups. Based on the pattern of the aromatic peaks, two doublets of doublets, we propose the polymer structure as indicated in Figure S1-A. The ${ }^{1} \mathrm{H}$ NMR spectra of $p$-phenylenediamine is represented by only one aromatic peak at $\delta_{\mathrm{H}} 6.3 \mathrm{ppm}$ (proton c). When the polymerization occurs, this peak decreases in intensity, and two new peaks are observed: one at $\delta_{\mathrm{H}} 5.9 \mathrm{ppm}$ (broad singlet) and the other at $\delta_{\mathrm{H}} 6.6 \mathrm{ppm}$ (doublet). These peaks lead us to propose the polymer structure of Figure S1-B.

The amount of oligomers and polymers produced by the different forms of laccase under high-pressure homogenization was evaluated by the weighting of the final powder obtained after the reaction. For both poly(catechol) and poly(p-phenylenediamine), the catalysis with PEGylated laccase and Epoxy-PEGylated laccase is enhanced independently on the enzyme container used. Comparing with the native form of laccase the conversion yield of catechol into poly(catechol) was improved up to $73 \%$ with PEGylated laccase, while the conversion of $p$ phenylenediamine into poly( $p$-phenylenediamine) was improved up to $85 \%$. These findings have been reported previously by us (data not shown) where we found that the chemically modified laccases are able to improve the polymerization of catechol. Moreover, the role of highenergy environments was also previously described as potentiating the enzymatic polymerization to produce higher polymers and convert higher amount of monomer [23,26]. A higher amount of produced oligomers and polymers not always corresponds to a higher deposition onto the fibre substrates used as containers. This may depend on the type of fibre and variations may occur as discussed further (Table 1).

\subsection{Surface characterization of coated fabrics}

We investigated the wettability surface properties of the fabrics by water contact angle analysis and by time of water drop absorption evaluation (Table 2). Cotton is a well-known hydrophilic natural polymeric material and when a droplet of water was placed on its surface, it was rapidly soaked into the fabric. After coating with both polymers, the water contact angle of cotton fabrics increased up to maximum $144.4^{\circ}$ and the time of water drop absorption increased from $0.1 \mathrm{~min}$. to over $5 \mathrm{~min}$., indicating an increase of the hydrophobicity. The hydrophobic character of PET was also incremented by coating with both poly(catechol) and poly(p-phenylenediamine) as it could be shown by the increase of the water absorption time and by the water contact angle. The wool fabric demonstrated however a distinct behaviour depending on the polymer. When coated with poly(catechol), an increase of hydrophobicity was observed whereas when coated poly( $p$ phenylenediamine) the hydrophobicity character decrease

Table 1

Conversion yield (\%) after polymerization of catechol and $p$-phenylenediamine with all laccase forms under high-pressure homogenization, using textile fabrics as enzyme containers (the error measured for all the samples was lower than $5 \%)$.

\begin{tabular}{|c|c|c|c|c|c|c|}
\hline \multirow{2}{*}{$\begin{array}{l}\text { Fabric } \\
\text { container }\end{array}$} & \multicolumn{3}{|c|}{ Poly(catechol)* } & \multicolumn{3}{|c|}{$\operatorname{Poly}(p \text {-phenylenediamine })^{*}$} \\
\hline & $\begin{array}{l}\text { Native } \\
\text { laccase }\end{array}$ & $\begin{array}{l}\text { PEGylated } \\
\text { laccase }\end{array}$ & $\begin{array}{l}\text { Epoxy- } \\
\text { PEGylated } \\
\text { laccase }\end{array}$ & $\begin{array}{l}\text { Native } \\
\text { laccase }\end{array}$ & $\begin{array}{l}\text { PEGylated } \\
\text { laccase }\end{array}$ & $\begin{array}{l}\text { Epoxy- } \\
\text { PEGylated } \\
\text { laccase }\end{array}$ \\
\hline PET & $60.8 \%$ & $72.8 \%$ & $63.0 \%$ & $72.8 \%$ & $84.8 \%$ & $71.0 \%$ \\
\hline Cotton & $61.2 \%$ & $69.8 \%$ & $67.6 \%$ & $77.2 \%$ & $83.8 \%$ & $79.6 \%$ \\
\hline Wool & $64.6 \%$ & $71.8 \%$ & $67.8 \%$ & $80.6 \%$ & 83.8 \% & $82.0 \%$ \\
\hline
\end{tabular}

* The conversion rates were calculated through samples weighing. 
Table 2

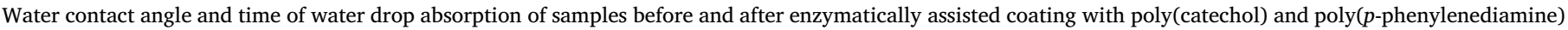
(values are the mean of 3 independent measurements).

\begin{tabular}{|c|c|c|c|c|c|}
\hline & & \multicolumn{2}{|l|}{ Poly(catechol) } & \multicolumn{2}{|c|}{ Poly(p-phenylenediamine) } \\
\hline & & $\begin{array}{l}\text { Water contact angle } \\
\left({ }^{\circ}\right)\end{array}$ & $\begin{array}{l}\text { Time of water drop absorption } \\
\text { (min.) }\end{array}$ & $\begin{array}{l}\text { Water contact angle } \\
\left({ }^{\circ}\right)\end{array}$ & $\begin{array}{l}\text { Time of water drop absorption } \\
\text { (min.) }\end{array}$ \\
\hline \multirow{4}{*}{ PET } & Control (No enzyme) & $89.3 \pm 1.14$ & 2.00 & $89.3 \pm 1.14$ & 2.00 \\
\hline & Native laccase & $130.4 \pm 0.57$ & 4.10 & $127.5 \pm 0.98$ & $>5.00$ \\
\hline & PEGylated laccase & $129.3 \pm 0.65$ & 4.40 & $131.8 \pm 0.56$ & $>5.00$ \\
\hline & Epoxy-PEGylated laccase & $131.1 \pm 1.14$ & $>5.00$ & $121.2 \pm 0.24$ & 2.50 \\
\hline \multirow[t]{4}{*}{ Cotton } & Control (No enzyme) & 0 & 0.10 & 0 & 0.10 \\
\hline & Native laccase & $136.8 \pm 1.30$ & $>5.00$ & $140.1 \pm 1.85$ & 3.70 \\
\hline & PEGylated laccase & $144.4 \pm 1.38$ & $>5.00$ & $140.9 \pm 1.77$ & 0.23 \\
\hline & Epoxy-PEGylated laccase & $142.9 \pm 1.85$ & $>5.00$ & 0 & 0.40 \\
\hline \multirow[t]{4}{*}{ Wool } & Control (No enzyme) & $*$ & $>5.00$ & * & $>5.00$ \\
\hline & Native laccase & * & $>5.00$ & * & 0.53 \\
\hline & PEGylated laccase & * & $>5.00$ & " & 0.60 \\
\hline & Epoxy-PEGylated laccase & $*$ & $>5.00$ & * & 1.30 \\
\hline
\end{tabular}

* The contact angle of wool could not be measured due to its surface morphology which did not permit to establish a baseline.

significantly. While that for PET and cotton it was observed that the coating remained mostly at the surface of the fabrics, for wool a deeper penetration of both polymers inside fibrils was perceptible. For this reason, this textile substrate did not acquired a hydrophobic behaviour. It is well-established that the water or humidity in the air exerts a significant influence on the charge decay and conductivity stability of conductive materials [31]. The good hydrophobicity of the fabrics would enhance their humidity resistance properties and suppress conductivity decay. This phenomenon was observed for both PET and cotton coated fabrics which revealed higher conductivity, while on wool was only possible to observe a small increment.

From the scanning electron microscopy (SEM) (Fig. 2), the changes of the micromorphology of coated fabrics are evident. The smooth surfaces of PET and cotton surfaces were found to be covered with both poly(catechol) and poly(p-phenylenediamine) layer. On wool, the covering was also confirmed by visualisation of the covering of the typical fibre scales. The overall coverage of the fabrics with the polymers is found to impart electrical conductivity to the fabrics, especially to PET and cotton. On wool, as mentioned before, the conductivity increment was not so perceptible maybe more due to a lower hydrophobicity than to the amount of polymer coated.

\section{3. "In situ" colouration of fabrics}

As we have previously highlighted, laccases have been applied for the "in situ" synthesis of colourant compounds, especially for protein and cotton fibres, however less explored for synthetic fibers [16,32,33]. Herein, we explore the "in situ" polymerization and colouration of textile fibres by placing the enzymes used inside a textile fabric bag with different fibre composition (PET, cotton and wool). The photographs of the colourized fabrics are shown in Table S1. Besides colouration, the newly polymers are expected to confer differentiated properties to the coated fabrics, namely electrical conductivity [15]. The spectral estimation of the textile fibers after "in situ" colouration is presented in Table 3 as checksum K/S quantification after a washing step with a non-ionic detergent. From the data obtained one might observe a deep colouration, especially of cotton and wool fabrics. The controls consisting on fabric samples incubated only with laccase were also conducted in order to evaluate the influence of the catalyst colour on the fabrics after homogenization. Insignificant colouration could be observed for these fabric controls.

Despite the lower coating, PET fibres are also colourized independently on the catalyst used. This corroborates our previous findings [23] where we studied for the first time the in situ enzymatic colouration of PET using native laccase. Herein, we confirm these results and deepen the study by using chemically modified laccases which incremented the polymerization. As expected, protein fibres like wool have great affinity to the newly polymers produced, as confirmed by the deep colouration obtained. From what concern poly( $p$-phenylenediamine), the role of the different laccase forms is not perceptible since high colouration is obtained for all. In the case of poly(catechol) the role of the different enzyme forms is more evident. As previously observed for the conversion rates, PEGylated enzyme promoted also the higher levels of colouration on cotton and wool. In the case of PET, this enzyme form contributes to a higher colouration with poly( $p$-phenylenediamine) but not with poly(catechol). Due to its hydrophobicity, lower affinity and lack of bonding with the formed polymers, the colouration of PET is still not uniform and no direct relation between the amount of polymers formed and fibres coverage might be established. However, despite the lower affinity observed for PET, the methodology applied revealed to be efficient not only for cotton and wool fibre colouration but also for synthetic substrates. The effect of "hot spots" and mass transport phenomena governed by high-pressure homogenization allowed to obtained high coverage of cotton and wool fibres also to colourize a fibre which naturally would not be prone to colourization. It is noteworthy that no additives were applied as in the traditional fabrics colouration. This promising technology showed potentialities for the green colouration of natural and synthetic fibres under mild reaction conditions, opening up an all new field of colouration where a panoply of polymers may be used as colourants. We foresee the possibility of the technology industrialization due to the great performance at industrial scale of the high-pressure homogenizers.

\subsubsection{Colour fastness after washing}

The colour fastness of coated samples with the colouring polymers produced was studied by washing the samples with a non-ionic detergent. The standard washing fastness procedure was not feasible due to the limited size of the samples. The colour difference between coated samples before and after washing is presented in Table 3 after grey scale evaluation by the software Datacolour spectra. The data obtained reveal acceptable colour fastness with exception of the coloured PET samples. This result was expectable given the low affinity of the polymers to this substrate. The other substrates, cotton and wool, being natural fibres with higher affinity to these compounds, present, as expected low colour loss after washing. No tendency is observed relating the colour loss with the type of enzyme used for the in situ polymerization. Apparently, the colour loss is more related to the substrate used rather than the ratio (oligomers: polymers) produced, which, considering our previous findings [23], are expected to be different depending on the enzyme formed used. One would expect a higher colour fastness of the samples coloured polymers produced by PEGylated laccase forms, 

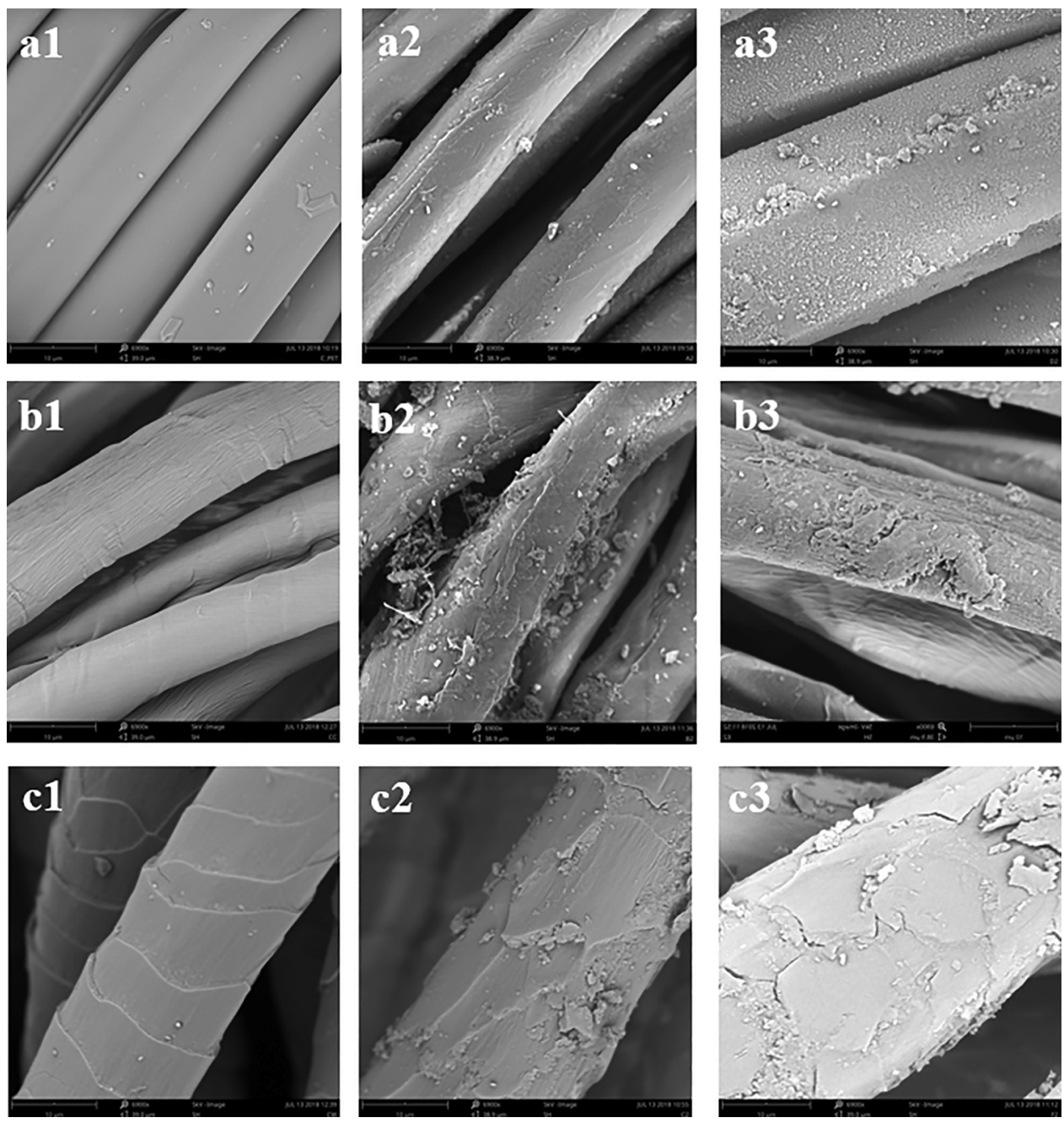

Fig. 2. SEM images after polymerization with PEGylated laccase: a1) control PET; a2) PET coated with poly(catechol); a3) PET coated with poly(p-phenylenediamine); b1) control cotton; b2) cotton coated with poly(catechol); b3) cotton coated with poly(p-phenylenediamine); c1) control wool; c2) wool coated with poly (catechol); c3) wool coated with poly(p-phenylenediamine).

which gave rise to higher amount of polymer. This tendency was not however observed.

In comparison with other related works, the coated fabrics reveal reasonable colour fastness to washing $[16,34]$. The mass transport effect promoted by the high-pressure homogenization is directly related with a higher substantivity of the new polymers onto the fabrics surface, which was confirmed in our previous study about the polymerization of catechol with native laccase using water bath reactor and high-pressure homogenizer reactor [23]. A higher polymer adhesion corresponds, in most cases studied, to a higher substantivity and low colour loss during washing process.

The colour fastness is a prime importance parameter especially for

Table 3

Checksum $(K / S)$ estimation of the colourized fabrics after in situ polymerization with native laccase, PEGylated laccase and Epoxy-PEGylated laccase under highpressure homogenization (before and after washing with non ionic detergent); the control samples were not coated with polymers.

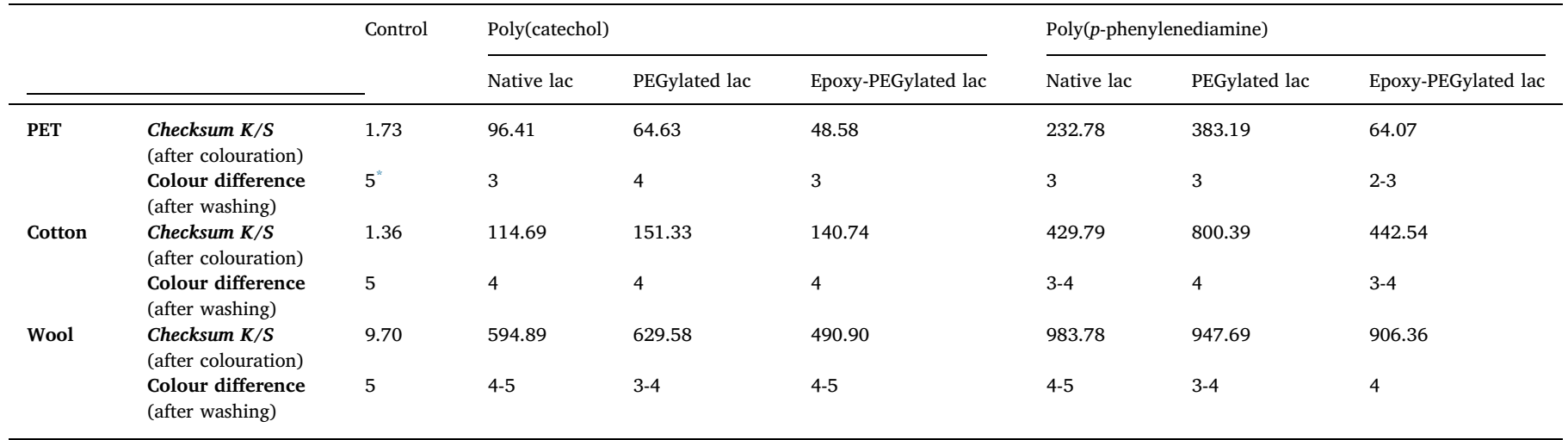

* 5: GOOD; 1: FAIR. 
A)
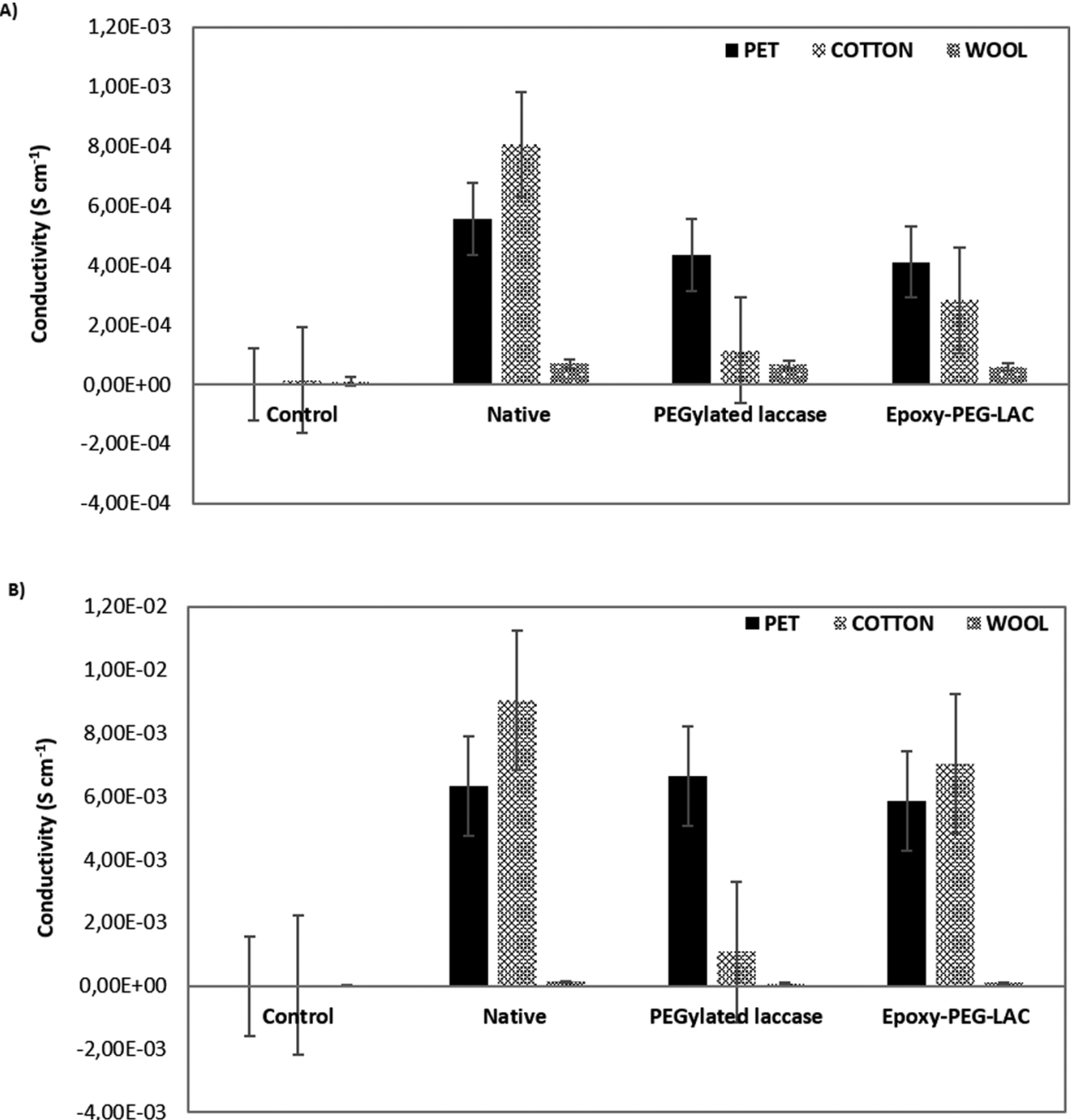

Fig. 3. Electric conductivity of fabrics coated with: a) poly(catechol) and a) poly(p-phenylenediamine) after in situ polymerization by laccase with native laccase, PEGylated laccase and Epoxy-PEGylated laccase.

daily-use garments, however if one consider the coated conductive fabrics as technical textiles, this parameter loses importance. Moreover, even after washing the samples maintained the conductivity character at similar levels (data not shown).

\subsection{Conductivity of poly(catechol) and poly(p-phenylenediamine) coated} fabrics

As mentioned previously, the coating of the fabrics within the newly formed oligomers/polymers is expected to confer additional properties to the substrates besides the colouration itself. Since the polymerization of both natural and artificial monomers with undivided electron pair goes through a radical mechanism, oxidoreductases like laccase, were used herein as biocatalysts for these reactions. The conductivity of the textile fibres after coating with poly(catechol) and poly(p-phenylenediamine) were evaluated (Fig. 3). When in monomer state these compounds present poor conductivity, acquiring higher conductivity when polymerized [35]. As can be depicted in Fig. 3A and B, the coated PET and cotton fabric samples with poly (catechol) and poly( $p$-phenylenediamine) varied from antistatic (control samples) to low conductive fabrics. The conductivity behaviour of coated wool is still negligible. The differences of conductivity between fabrics might be due to several reasons. Despite the similar conversions rates observed, the different level of polymer penetration into the fibres may influence greatly the surface conductivity. In the case of wool, one could observe a higher colouration, as seen by checksum $K / S$, but deeper colour penetration (data not shown), which might influence the amount of polymer at the surface of the fabric able to act as electricity conductor.

The conductivity of fabrics coated with poly( $p$-phenylenediamine) is higher than the fabrics coated with poly(catechol) (Fig. 3B). The electric conductivity of both materials is related with the delocalization of the $\pi$-electron systems with alternating single and double bonds, which can be easily oxidized and reduced. The final structure of poly(p-phenylenediamine) containing fused aromatic rings [36], in comparison with the poly(catechol), allows a higher electron delocalization which might be responsible for the conductivity differences obtained.

\section{Conclusions}

We successfully coated textile fabrics (PET, cotton and wool) with poly(catechol) and poly(p-phenylenediamine) to obtain coloured fabrics with additional conductive properties. The in situ oxidation of catechol and $p$-phenylenediamine with native and chemically modified laccases, properly contained inside fabric bags which had served simultaneously as enzyme containers and substrates for coating, resulted in a green colouration of these fabrics. The fixation of the polyphenols to the textile substrates was incremented, relatively to other works reported, due to the inherent mass transport phenomena involved within the high-energy environment applied. Besides colouration, the polymers deposited conferred electric conductive properties to the coated fabric substrates. One can also highlight that this additional property is not directly related to the form of enzyme used for oxidation 
nor to the amount of polymers deposited at the fibre's surface, but is yet associated with the type of starting monomer. It is clear that a higher conductivity is obtained after coating with poly( $p$-phenylenediamine), the product of $p$-phenylenediamine oxidation, which is in theory more conductive than catechol.

The promising technology herein presented demonstrates potentialities for the green colouration of natural and synthetic fibres under mild reaction conditions, opening up new routes for colouration where a panoply of polymers may be used as colourants.

\section{Declaration of interest statement}

The authors declare no competing financial and non-financial interests.

\section{Acknowledgements}

This study was supported by the Chinese Government Scholarship under China Scholarship Council [grant No. 201606790036]; and by the Chinese Foundation Key projects of governmental cooperation in international scientific and technological innovation [grant No. 2016 YFE0115700]; and by the Portuguese Foundation for Science and Technology (FCT) under the scope of the strategic funding of UID/BIO/ 04469/2013 unit and COMPETE 2020 (POCI-01-0145-FEDER-006684) and BioTecNorte operation (NORTE-01-0145-FEDER-000004) funded by European Regional Development Fund under the scope of Norte2020 - Programa Operacional Regional do Norte.

This work was also supported by the European Union through the European Regional Development Fund (ERDF) under the Competitiveness Operational Program [grant COP-A1.1.4-E-2015nr.30/ 01.09.2016]; and by the Project "Search-ON2: Revitalization of HPC infrastructure of UMinho" [grant NORTE-07-0162-FEDER-000086), cofunded by the North Portugal Regional Operational Programme (ON.2 O Novo Norte), under the National Strategic Reference Framework (NSRF), through the European Regional Development Fund (ERDF).

The work was also supported by the FCT- Fundação para a Ciência e a Tecnologia [grant SFRH/BD/121673/2016 and IF/00186/2015].

\section{References}

[1] M.M. Omar, Phenolic compounds in botanical extracts used in foods, flavors, cosmetics, and pharmaceuticals, phenolic compounds in food and their effects on health I, Am. Chem. Soc. (1992) 154-168.

[2] T. Hu, R.E. Bailey, S.W. Morrall, M.J. Aardema, L.A. Stanley, J.A. Skare, Dermal penetration and metabolism of p-aminophenol and p-phenylenediamine: application of the EpiDerm ${ }^{\mathrm{TM}}$ human reconstructed epidermis model, Toxicol. Lett. 188 (2) (2009) 119-129.

[3] S. Cohen, P.A. Belinky, Y. Hadar, C.G. Dosoretz, Characterization of catechol derivative removal by lignin peroxidase in aqueous mixture, Bioresour. Technol. 100 (7) (2009) 2247-2253.

[4] K. Ikehata, J.A. Nicell, Characterization of tyrosinase for the treatment of aqueous phenols, Bioresour. Technol. 74 (3) (2000) 191-199.

[5] A. Bassi, Z. Geng, M. Gijzen, Enzymatic removal of phenol and chlorophenols using soybean seed hulls, Eng. Life Sci. 4 (2) (2004) 125-130.

[6] N. Aktaş, N. Şahiner, Ö. Kantoğlu, B. Salih, A. Tanyolaç, Biosynthesis and characterization of laccase catalyzed poly(Catechol), J. Polym. Environ. 11 (3) (2003) 123-128.

[7] J. Polak, A. Jarosz-Wilkolazka, Fungal laccases as green catalysts for dye synthesis, Process Biochem. 47 (9) (2012) 1295-1307.

[8] E. Acero, T. Kudanga, A. Ortner, I. Kaluzna, S. de Wildeman, G. Nyanhongo, G. Guebitz, Laccase functionalization of flax and coconut fibers, Polymers 6 (6) (2014) 1676-1684.
[9] M. Božič, J. Štrancar, V. Kokol, Laccase-initiated reaction between phenolic acids and chitosan, React. Funct. Polym. 73 (10) (2013) 1377-1383.

[10] G. Shumakovich, V. Kurova, I. Vasileva, D. Pankratov, G. Otrokhov, O. Morozova, A. Yaropolov, Laccase-mediated synthesis of conducting polyaniline, J. Mol. Catal. B-Enzym. 77 (2012) 105-110.

[11] C. Fleischmann, M. Lievenbrück, H. Ritter, Polymers and dyes: developments and applications, Polymers 7 (4) (2015) 717-746.

[12] H. Shin, G. Guebitz, A. Cavaco-Paulo, "In situ" enzymatically prepared polymers for wool coloration, Macromol. Mater. Eng. 286 (11) (2001) 691-694.

[13] T. Tzanov, C.J. Silva, A. Zille, J. Oliveira, A. Cavaco-Paulo, Effect of some process parameters in enzymatic dyeing of wool, Appl. Biochem. Biotechnol. 111 (1) (2003) $1-13$.

[14] S. Kim, C. Lopez, G. Güebitz, A. Cavaco-Paulo, Biological coloration of flax fabrics with flavonoids using laccase from Trametes hirsuta, Eng. Life Sci. 8 (3) (2008) 324-330.

[15] S. Kim, A. Cavaco-Paulo, Laccase-catalysed protein-flavonoid conjugates for flax fibre modification, Appl. Microbiol. Biotechnol. 93 (2) (2012) 585-600.

[16] S. Kim, D. Moldes, A. Cavaco-Paulo, Laccases for enzymatic colouration of unbleached cotton, Enzyme Microb. Technol. 40 (7) (2007) 1788-1793.

[17] J.R. Jeon, P. Baldrian, K. Murugesan, Y.S. Chang, Laccase-catalysed oxidations of naturally occurring phenols: from in vivo biosynthetic pathways to green synthetic applications, Microb. Biotechnol. 5 (3) (2012) 318-332.

[18] G.S. Nyanhongo, T. Kudanga, E.N. Prasetyo, G.M. Guebitz, Enzymatic polyme functionalisation: advances in laccase and peroxidase derived lignocellulose functional polymers, in: G.S. Nyanhongo, W. Steiner, G.M. Gubitz (Eds.), Biofunctionalization of Polymers and Their Applications, 2011, pp. 47-68.

[19] G.V. Otrokhov, O.V. Morozova, I.S. Vasil'eva, G.P. Shumakovich, E.A. Zaitseva, M.E. Khlupova, A.I. Yaropolov, Biocatalytic synthesis of conducting polymers and prospects for its application, Biochem.-Moscow 78 (13) (2013) 1539-1553.

[20] T. Zhang, R. Bai, J. Shen, Q. Wang, P. Wang, J. Yuan, X. Fan, Laccase-catalyzed polymerization of diaminobenzenesulfonic acid for $\mathrm{pH}$-responsive color-changing and conductive wool fabrics, Text. Res. J. (2017).

[21] Z. Ya, D. Aixue, F. Xuerong, W. Qiang, Z. Ying, Y. Yuanyuan, C.-P. Artur, Laccasecatalyzed synthesis of conducting polyaniline-lignosulfonate composite, J. Appl. Polym. Sci. 133 (5) (2016).

[22] F. de Salas, I. Pardo, H.J. Salavagione, P. Aza, E. Amougi, J. Vind, A.T. Martinez, S. Camarero, Advanced synthesis of conductive polyaniline using laccase as biocatalyst, PLoS One 11 (10) (2016).

[23] J. Su, T.G. Castro, J. Noro, J. Fu, Q. Wang, C. Silva, A. Cavaco-Paulo, The effect of high-energy environments on the structure of laccase-polymerized poly(catechol), Ultrason. Sonochem. 48 (2018) 275-280.

[24] D. Campaniello, A. Bevilacqua, M. Sinigaglia, M.R. Corbo, Using homogenization, sonication and thermo-sonication to inactivate fungi, PeerJ 4 (2016).

[25] M.T.K. Kubo, P.E.D. Augusto, M. Cristianini, Effect of high pressure homogenization (HPH) on the physical stability of tomato juice, Food Res. Int. 51 (1) (2013) 170-179.

[26] J. Su, J. Noro, A. Loureiro, M. Martins, N.G. Azoia, J. Fu, Q. Wang, C. Silva, A. Cavaco-Paulo, PEGylation greatly enhances laccase polymerase activity, ChemCatChem 9 (20) (2017) 3888-3894.

[27] S.M. Daly, T.M. Przybycien, R.D. Tilton, Adsorption of poly(ethylene glycol)Modified lysozyme to silica, Langmuir 21 (4) (2005) 1328-1337.

[28] R.E. Childs, W.G. Bardsley, The steady-state kinetics of peroxidase with 2,2'-azinodi-(3-ethyl-benzthiazoline-6-sulphonic acid) as chromogen, Biochem. J. 145 (1) (1975) 93-103.

[29] T. Tzanov, M. Calafell, G.M. Guebitz, A. Cavaco-Paulo, Bio-preparation of cotton fabrics, Enzyme Microb. Technol. 29 (6) (2001) 357-362.

[30] P.K. Jha, G.P. Halada, The catalytic role of uranyl in formation of polycatechol complexes, Chem. Cent. J. 5 (1) (2011) 12.

[31] B. Wu, B. Zhang, J. Wu, Z. Wang, H. Ma, M. Yu, L. Li, J. Li, Electrical switchability and dry-wash durability of conductive textiles, Sci. Rep. 5 (2015) 11255.

[32] K. Li, F. Xu, K.-E.L. Eriksson, Comparison of fungal laccases and redox mediators in oxidation of a nonphenolic lignin model compound, Appl. Environ. Microbiol. 65 (6) (1999) 2654-2660.

[33] C.D. Blanco, M.D. González, J.M.D. Monmany, T. Tzanov, Dyeing properties, synthesis, isolation and characterization of an in situ generated phenolic pigment, covalently bound to cotton, Enzyme Microb. Technol. 44 (6-7) (2009) 380-385.

[34] K. Suyeon, L. Hyunkyung, K. Juhea, O. Fernando, S. Pedro, K. Hyerim, N. Javier, Laccase-mediated grafting of polyphenols onto cationized cotton fibers to impart UV protection and antioxidant activities, J. Appl. Polym. Sci. 135 (6) (2018) 45801.

[35] L.M. Mansour, Z.E. Nazarzadeh, M.P. Najafi, Synthesis of novel conductive poly (p-phenylenediamine)/ Fe3O4 nanocomposite via emulsion polymerization and investigation of antioxidant activity, Adv. Polym. Technol. 33 (1) (2014).

[36] F. Cataldo, On the polymerization of P-phenylenediamine, Eur. Polym. J. 32 (1) (1996) 43-50. 